\title{
Confección por dos filarias (Loa loa y Mansonella perstans) en una paciente proveniente de Guinea
}

\section{Loa Loa and Mansonella perstans coinfection in a patient from Guinea}

\author{
A. Gil-Setas ${ }^{1}$, M. Pérez Salazar², A. Navascués', F. Rodríguez Eleta² ${ }^{2}$ J.A Cebamanos ${ }^{3}$, \\ M.T. Rubio ${ }^{4}$
}

\section{RESUMEN}

Presentamos un caso de una infección mixta por 2 filarias (Loa loa y Mansonella perstans). La infección se sospechó, al aparecer, una marcada eosinofilia en una analítica de rutina. En la extensión de sangre periférica se observaban microfilarias envainadas y sin envainar, con lo que se estableció un diagnóstico de infección mixta por filarias. El diagnóstico definitivo de especie se realizó en el Centro Nacional de Microbiología de Majadahonda. Se instauró un tratamiento con dietilcarbamicina y mebendazol que conllevó la curación de los síntomas de la paciente y la eliminación de las microfilarias en sangre.

La filariasis es todavía endémica en muchos países. Debido al aumento de viajeros a dichas zonas y a los movimientos migratorios desde dichas áreas, no es infrecuente enfrentarnos a su diagnóstico. La implantación de tratamientos masivos contra las filarias en zonas endémicas por parte de la Organización Mundial de la Salud está disminuyendo su transmisión, consiguiendo la eliminación de la enfermedad en algunas áreas.

Palabras clave. Loa loa. Mansonella perstans. Filaria.

\begin{abstract}
We present a case of mixed infection by two filariae (Loa loa and Mansonella perstans). It seems that the infection was suspected due to a pronounced eosinophilia in a routine analysis. Sheathed and unsheathed microfilariae were observed in the spread of peripheral blood, which enabled a diagnosis to be established of mixed infection by filariae. The definitive diagnosis of the species was carried out at the National Centre of Microbiolgy of Majadahonda. A treatment was initiated with dietilcarbamazina and mebendazole which resulted in the patient's being cured and in the elimination of the microfilariae in the patient's blood.

Filariasis is still endemic in many countries. Due to the increase of travellers to such zones and the migratory movements from such areas it is not unusual for us to come across such a diagnosis. The implantation of massive treatments against filariae in endemic zones by the World Health Organisation is reducing their transmission and is managing to eliminate the disease in some areas.
\end{abstract}

Key words. Loa loa. Mansonella perstans. Filaria.
1. Servicio de Microbiología. Servicio Navarro de Salud-Osasunbidea.

2. Servicio de Hematología. Servicio Navarro de Salud-Osasunbidea.

3. Servicio de de Medicina de Familia. Servicio Navarro de Salud-Osasunbidea.

4. Servicio de Medicina Interna. Servicio Navarro de Salud-Osasunbidea.

\author{
Correspondencia \\ Alberto Gil-Setas \\ Servicio de Microbiología \\ Hospital de Navarra \\ Irunlarrea, 3 \\ 31008 Pamplona \\ E-mail: agilseta@navarra.es
}




\section{INTRODUCCIÓN}

Las filarias son nematodos (gusanos redondos) pertenecientes a dos familias, la familia Filariidae y la familia Dracunculidae. Coloquialmente a los miembros de ambas familias se les conoce como filarias debido a que son gusanos de cuerpo fino "filariforme», a pesar de existir importantes diferencias biológicas y de tratamiento entre amba. Aunque existen alrededor de 200 filarias parásitas, solamente unas pocas parasitan al hombre. Los adultos de la familia Filariidae se encuentran en el sistema linfático, tejido subcutáneo o tejido conectivo profundo. La hembra adulta produce microfilarias (estados larvarios) móviles que pueden encontrarse, dependiendo de las especies, en sangre periférica o en la piel. Las filarias humanas son transnmitidas por artrópodos vectores hematófagos ${ }^{1}$. Según la Organización Mundial de la Salud (OMS), alrededor de 752 millones de personas viven en países donde la filariasis es endémi- ca y se calcula que 80 millones de personas visitan al año estos países ${ }^{2}$.

Cada especie de filaria patógena para el hombre tiene una distribución geográfica concreta: Loa loa, es endémica en las zonas húmedas de África occidental y África central, mientras que Mansonella perstans se distribuye en toda África central y noreste de Sudamérica ${ }^{1}$.

\section{CASO CLÍNICO}

Paciente de 69 años de edad procedente de Guinea que llevaba un mes en España. Acudió a su médico de atención primaria por presentar desde hace un tiempo un cuadro de tos con expectoración, malestar general, mialgias, prurito intenso y fiebre (que no se objetiva en la consulta). La exploración física es normal. Se remiten muestras al laboratorio para bioquímica básica y hemograma. Sólo son destacables una ligera anemia 11,3 g/dL de hemoglobina y eosinofilia $2,6^{*} 1.000 / \mathrm{ml}$. En la revisión de la extensión de sangre teñida con Giemsa se observaron microfilarias envainadas (Fig. 1).

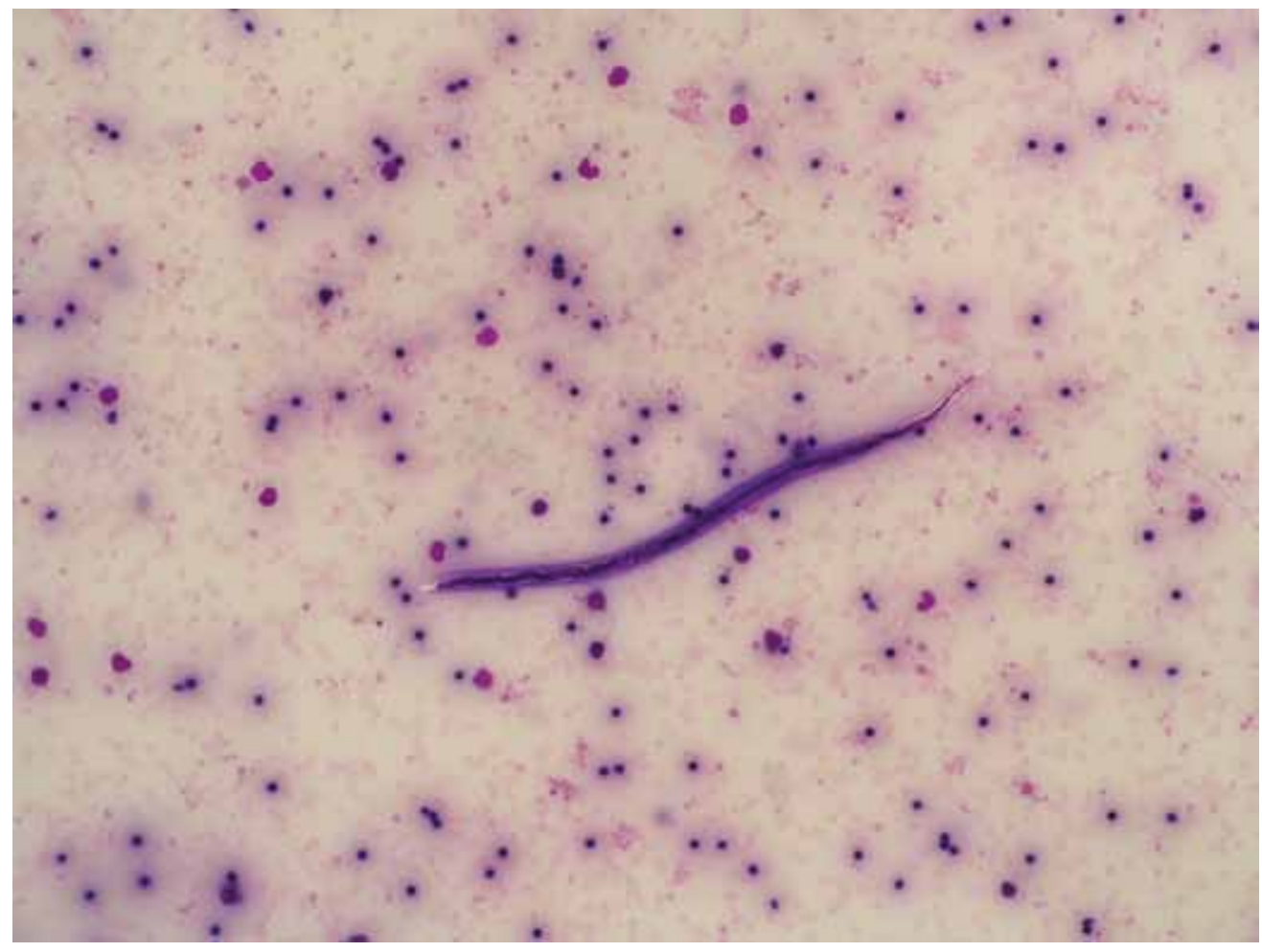

Figura 1. Microfilaria envainada: Loa loa. 
Dadas las diferencias de periodicidad de aparición de las microfilarias en sangre periférica (diurna y nocturna), se decidió ingresar a la paciente para tomar una segunda muestra de sangre que confirmase el diagnóstico de filaria- sis. En la segunda muestra de sangre periférica, tras concentración de Knott y tinción con Giemsa, se observaron microfilarias envainadas y sin envainar (Fig. 2).

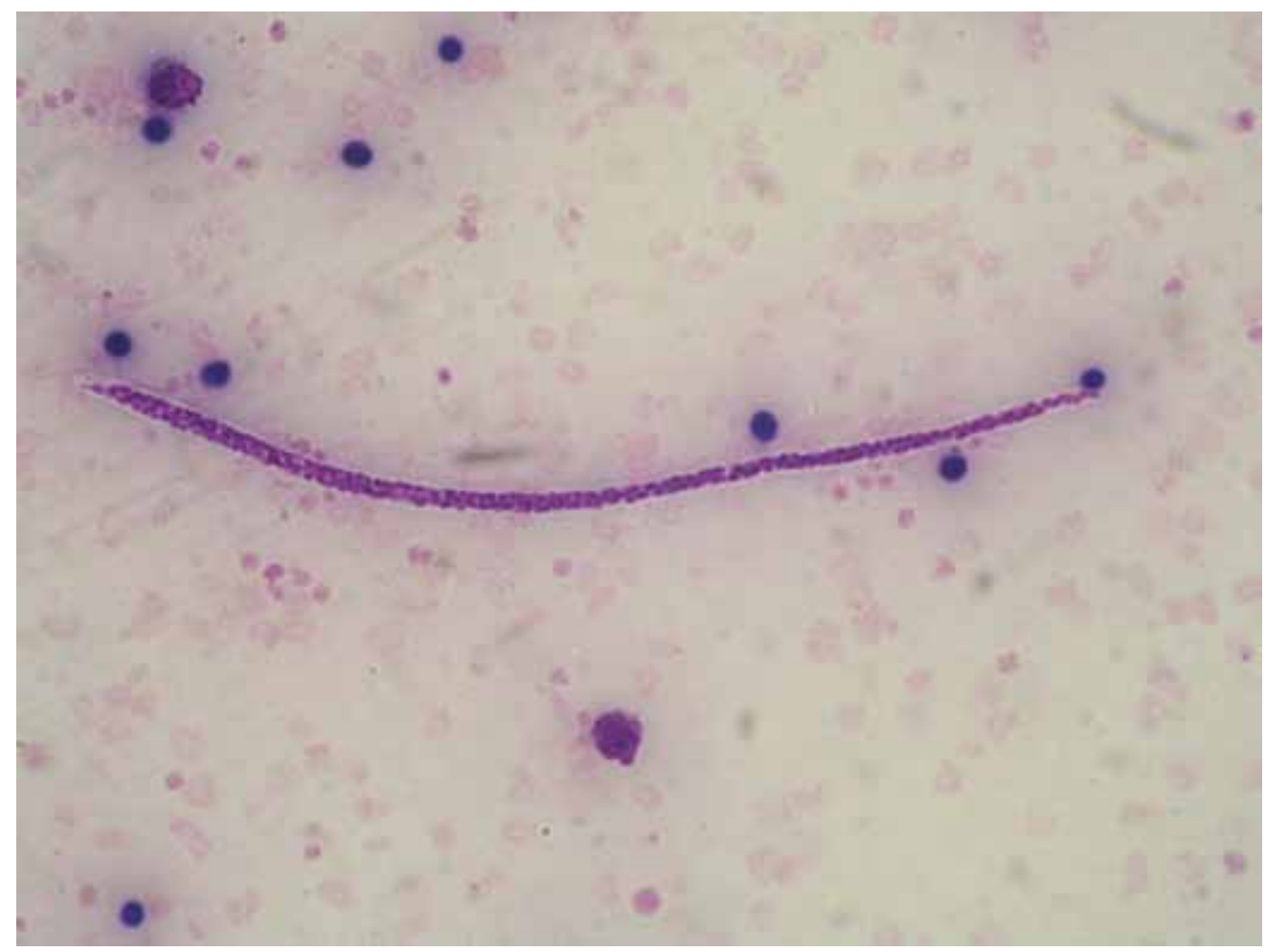

Figura 2. Microfilaria sin envainar: Mansonella perstans.

Se remitió la muestra al Centro Nacional de Microbiología, donde se identificaron como Loa loa y Mansonella perstans. Se inició un tratamiento con dietilcarbamicina $6 \mathrm{mg}$ en dosis única y $100 \mathrm{mg}$ de mebendazol cada 12 horas durante 30 días bajo supervisión médica los primeros días en previsión de una posible reacción de Herxheimer. La evolución fue buena, con disminución de la cifra de eosinófilos, mejoría del prurito y desaparición de las microfilarias en sangre periférica.

\section{DISCUSIÓN}

El diagnóstico de la filariasis se basa en la sospecha clínica ante pacientes que han viajado o que provienen de países donde la enfermedad es endémica. Los pacientes con filariasis pueden presentar una clínica aguda florida que incluye edemas, dolor de cabeza, fiebre, nódulos y prurito (dependiendo de la especie de filaria), una clínica crónica con linfedema, ceguera, etc. o ser una infección asintomática con eosinofilia en sangre periférica. En los pacientes con eosinofilia (más de 600 eosinófilos/ml), es importante investigar el antecedente de viajes a zonas endémicas de ciertas infecciones, incluyendo infecciones por helmintos. El diagnóstico microbiológico se basa fundamentalmente en la visualización del parásito en diferentes muestras (sangre periférica, piel...). 
También se utiliza la amplificación de ácidos nucleicos y la detección de antígenos circulantes.

Loa loa es una filaria que en la forma adulta vive en tejido subcutáneo, con microfilarias de periodicidad diurna en sangre periférica, que es cuando son transmitidas por los vectores (insectos hematófagos de hábitos diurnos). Su distribución se restringe a la zona oeste y central de África. Con frecuencia, la infección por Loa loa es asintomática y sólo se descubre la microfilaremia después del hallazgo de un adulto migrando por la conjuntiva ocular. Las manifestaciones clínicas más típicas incluyen el edema de Calabar y las manifestaciones alérgicas, que son más frecuentes en los viajeros en zonas endémicas que en la población autóctona.

Mansonella perstans reside en cavidades corporales como la peritoneal, pleural o pericárdica, encontrándose las microfilarias en sangre sin periodicidad. Mansonella perstans se distribuye por África central y el noreste de Sudamérica. La mayoría de infecciones son asintomáticas pero entre los casos sintomáticos encontramos angioedema, prurito, fiebre y dolor de cabeza ${ }^{1}$.

Se ha sugerido que la infección por filarias requiere exposiciones intensas y prolongadas a los vectores que las transmiten. Sin embargo, en un estudio del GeoSentinel Surveillance Network con 43.722 viajeros de zonas endémicas se ha visto que el tiempo de exposición no jugaba un papel clave en el porcentaje de infecciones (30\% para viajes de menos de 30 días de duración y $38 \%$ para viajes de más de 180 días), sino que el uso de repelentes, mosquiteras para las camas y la proximidad a las zonas donde habita el vector serían las causas del mayor o menor riesgo de infección. Comparativamente, los visitantes de países donde la filariasis no es endémica y que son infectados por filarias, presentan con mayor frecuencia e intensidad síntomas clínicos ${ }^{2}$.

Es frecuente entre los habitantes de zonas endémicas la parasitación con varias especies diferentes de filarias ${ }^{3}$.
El tratamiento y los programas de erradicación de la filariasis se basan en 3 fármacos:

1. La dietilcarbamicina, (DEC) es un fármaco con acción frente a las microfilarias y al adulto. El tratamiento recomendado para la DEC es una dosis única de $6 \mathrm{mg} / \mathrm{kg}$ que es tan efectiva como la pauta clásica de 12 días. No posee casi efectos adversos directos; el mayor efecto secundario se debe a la rápida destrucción de microfilarias en sangre.

2. La ivermectina no posee actividad frente a los adultos pero sí frente a las microfilarias. Se utiliza en dosis única de $200-400 \mu \mathrm{gm} / \mathrm{kg}$. Sus efectos secundarios derivados de la destrucción sanguínea de las microfilarias son más leves que los producidos por DEC ya que el aclaramiento de la parasitemia es más lento. Sin embargo, se describe una encefalopatía por Loa loa en tratamientos de pacientes coinfectados con Oncocerca y Loa loa con alta parasitemia de microfilarias de Loa loa en sangre ${ }^{4,5}$.

3. El albendazol es un antihelmíntico que actúa frente al adulto a dosis de $400 \mathrm{mg}$ dos veces al día en pauta de 2 semanas. No tiene acción frente a las microfilarias.

Los tres fármacos se utilizan en los programas de eliminación global de la filariasis. Las dosis únicas anuales de DEC o ivermectina son eficaces en prevenir la transmisión de la enfermedad al disminuir de manera sustancial y por tiempo prolongado el número de microfilarias en sangre. Cuando se asocia albendazol a DEC o ivermectina, la destrucción de microfilarias es todavía mayor ${ }^{6}$.

Otros fármacos usados en el tratamiento de la filariasis son: suramina, metrifonato, mebendazol, flubendazol, thiourea y tetraciclinas. El mebendazol es el tratamiento de elección en Mansonella perstans a dosis de $100 \mathrm{mg} / 8 \mathrm{~h}$ durante 30 días, ya que la ivermectina es poco eficaz frente a esta filaria. En el año 2007 había 48 países implementando programas de erradicación de la filariasis lin- 
fática, lo que supone una población de 752 millones de personas. Desde que se iniciaron estos programas se han administrado más de 1 billón de tratamientos, que han conducido a la reducción significativa de la presencia e intensidad de la microfilaremia y a prevenir la infección en millones de niños ${ }^{5}$.

La infección puede ser tratada con antifiláricos, aunque los síntomas crónicos derivados de la infección requerirán otro tipo de medidas como la cirugía para el hidrocele o el cuidado de la piel y ejercicio para mejorar los síntomas del linfedema.

\section{BIBLIOGRAFÍA}

1. McPherson T, Nutman T. Filarial nematodes. Manual of Clinical Microbiology, 2 volumen set, 9th Edition 2007. ASM Press Washington, DC 20036-2904.
2. LipNer EM, LaW MA, Barnett E, Keystone JS, Von SonNenburg F, Loutan L et al. Filariasis in travelers presenting to the Geosentinel Surveillance Network. PLoS Negl Trop Dis 1: e88.

3. Wanji S, Tendongfor N, Esum M, Ndineng S, ENYONG P. Epidemiology of concomitant infections due to Loa Loa, Mansonella perstans, and Onchocerca volvulus in rain forest villages of Cameroon. Med Microbiol Inmunol 2003; 192: 15-21.

4. DuKE BO. Overview: report of a scientific working group on seious adverse events following mectizan treatment of onchocerciasis in Loa Loa endemic areas. Filaria J 2003, 2 (Suppl. 1): SI.

5. Global programme to eliminate lymphatic filariasis. Worl Health Organization. Weekly epidemiological record 2008; 37/38: 333-348.

6. Palumbo E. Filariasis: diagnosis, treatment and prevention. Acta Biomed 2008; 79:106109 . 
